\title{
Preferential access to emotion under attentional blink: evidence for threshold phenomenon
}

\begin{abstract}
The present study provides evidence that the activation strength produced by emotional stimuli must pass a threshold level in order to be consciously perceived, contrary to the assumption of continuous quality of representation. An analysis of receiver operating characteristics (ROC) for attentional blink performance was used to distinguish between two (continuous vs. threshold) models of emotion perception by inspecting two different ROC's shapes. Across all conditions, the results showed that performance in the attentional blink task was better described by the two-limbs ROC predicted by the Krantz threshold model than by the curvilinear ROC implied by the signal-detection theory.

Key words: awareness, threshold model, attentional blink, emotional representation, stream of rapidly changing distractors (RSVP)
\end{abstract}

\section{INTRODUCTION}

There is commonplace finding that emotional representations tend to have preferential access to awareness. Invisible affective stimuli such as fearful facial expressions under conditions of continuous flash suppression, for example, reach awareness faster than non-emotional stimuli (Yang, Zald, \& Blake, 2007). Experiments of emotional processing in an attentional blink paradigm led to similar conclusions: emotional stimuli were strong enough to result in awareness unlike typical, non-emotional targets that fail to enter awareness even though the attentional blink conditions suggested that they should be consciously reported (Shapiro, Raymond, \& Arnell, 1997). Particularly, in the attentional blink task when two non-emotional targets (so called T1 and T2 stimuli) are masked in the stream of rapidly changing distractors (RSVP) and presented sequentially within a short interval of 200-500 ms, participants are often unable to report T2 accurately, even though T1 was correctly reported. However, performance of detecting T2 improves dramatically when the targets are emotional. Milders, Sahraie, Logan, and Donnelon (2006) ran the attentional blink paradigm with emotional faces and demonstrated that there was enhanced detection of the emotional faces and T2 were not "blinked". Similarly, emotionally meaningful stimuli such as fearful faces (Stein, Peelen, Funk, \& Seidl, 2010), or schematic angry and happy faces (Maratos, Mogg, \& Bradley, 2008) were less affected by the attentional blink than were neutral stimuli. It is important therefore to establish what factors determine if emotional input receives higher priority and increased attention that enables T2 to reach the level of awareness sufficient for reporting.

One reason for this effect might be that the emotional representation to which subjects claim to have conscious access has some unique quality. That is, the function relating the activation strength of emotional input to awareness may contain thresholds, contrary to currently popular signal detection models whose perceptual representations are on a continuum (Kanwisher, 2001). Evidence from the attentional blink task with a continuous rating scale (Sergent \& Dehaene, 2004) provides support for the notion that conscious awareness may operate in the "allor-none" manner. Their attentional blink procedure resulted in the bimodal distributions of confidence with two distinct single peaks, one at $100 \%$ visibility for T2-present trials, and the other one at $0 \%$ visibility for $\mathrm{T} 2$-absent trials. Thus,

\footnotetext{
* Faculty in Wroclaw, University of Social Sciences and Humanities, Poland

** Key Laboratory of Adolescent Cyberpsychology and Behavior, (Central China Normal University), Ministry of Education, China

*** Department of Psychology and Neuroscience, University of Colorado, USA

$* * * *$ University of Social Sciences and Humanities, Faculty in Wroclaw, 30b Ostrowskiego Street, 53-238 Wroclaw, Poland; rszczepanowski@swps.edu.pl
} 
the participants used the scale in the "all-or-none" manner even though it was designed to be sensitive to continuous changes of perception.

According to a signal-detection theory (SDT) framework, activation strength underlying observer's decision in the above-mentioned attentional blink paradigm with the confidence judgments task by Sergent and Dehaene (2004) should be supported by an "all-or-none" dimension rather than a continuous dimension (Green \& Swets, 1966). Under the classical signal-detection theory, the distributions of internal signal strength underlying decision of a continuous dimension are Gaussian, and lead to a curvilinear shape of the ROC curve (Macmillan \& Creelman, 2005) as shown in Fig.1A. The threshold model of perception posits that decision space has "all-or none" (discrete) property that are observer's thresholds that lead to rectangular distributions with different heights (Macmillan \& Creelman, 2005). Following a three-state low- and highthreshold (3-LHT) model by Krantz (1969), the ROC consists of two limbs (lower and upper) of different slopes (see Fig.1B). The shape of the threshold ROC curve is in fact linear indicating that a linear increase in hit and false alarm rates happen with progressively more lenient criterion placement (Slotnick \& Dodson, 2005).

The present study used the ROC analysis of the emotional attentional blink to provide an empirical support for the hypothesis that activation strength to awareness for the emotionally meaningful items contains thresholds. The $\mathrm{AB}$ data were used to generate ROC curves to be fit with the threshold and the signal-detection models. Better fits obtained with the threshold model would indicate that the function relating activation strength of emotional representation to awareness occurs in the "all-or-none" fashion. Whereas the curvilinear shape ROC by the signaldetection model would tell us that this function should rather vary along continuous dimension.

\section{METHOD}

\section{Participants}

Thirteen right-handed students (6 women and 7 men) from Warsaw School of Social Sciences and Humanities participated in the study for credit points, and agreed to give an informed consent to participate. All subjects had correct or corrected-to-normal vision. The study was approved by the Ethics Committee of the School. Dataset from one subject was removed due to failure in using the confidence ratings scale.

\section{Procedure}

The present study replicated the AB paradigm using emotional faces by Milders et al. (2006) with an important modification concerning to a confidence measure of T2 visibility. A Rapid Serial Visual Presentation (RSVP) paradigm was used in this experiment (see Fig.1C). Each RSVP trial was composed of 22 items of either targets (emotional faces) or distractors (scrambled faces). Faces stimuli were selected from the pool of standardized emotional expressions sets (Ekman \& Friesen, 1976; Lundqvist, Flykt, \& Öhman, 1998). All stimuli ware presented on the 22" Iiyama MA203DT VisionMaster Pro 513 monitor (a screen refresh rate of $120 \mathrm{~Hz}$ ) driven by the ATI Radeon HD 4800Series graphics card. The viewing distance was approximately $50 \mathrm{~cm}$, and face stimuli subtended $4^{\circ} \times 5^{\circ}$ of visual angle. Half of trials contained two targets (both T1 and T2) while the other half only contained one target (only T1). The 1st target was always a neutral face. Emotional T2 was a happy or fearful face with equal chance. For the T2-absent trials, a scrambled face was used. We used the scrambled face as a non-emotional foil to avoid any potential confusion in perceiving a neutral facial expression as being emotionally charged as reported elsewhere (Phillips et al., 2001; Thomas et al., 2001). Each RSVP item was presented for $80 \mathrm{~ms}$, and replaced immediately by the next item. The first target (T1) appeared between items of 8-12 within the trial. A T1-T2 lag was $160 \mathrm{~ms}$ (lag 2), $240 \mathrm{~ms}$ (lag 3), or $320 \mathrm{~ms}$ (lag 4). The twenty-two different scrambled faces were produced by rearranging the internal features of two neutral faces (one male and one female). T1 was selected from the pool of 16 faces of neutral expression ( 8 males and 8 females of different identities). T2 was selected from the pool of 8 faces of different identities, including a half of fearful expression ( 2 males and 2 females) and half of happy expression ( 2 males and 2 females). Thus, the identities of T1 were never repeated as the identities in T2, both within a single trial and across different trials. At the same time, the factors of gender and identity were counterbalanced for both T1 and T2. Subjects performed 576 trials in a random order, which followed a 3 (the T1-T2 lag: lag 2, lag 3, lag 4) by 2 (the expression: emotional expression, no expression) design. Trials from different conditions were interleaved in random order, and presented in 6 different blocks of the equal trial number.

At the beginning of each trial, participants were instructed to fixate at the center of the display. After a $750 \mathrm{~ms}$ blank interval, the RSVP items were presented sequentially in the same location. Immediately, after the RSVP sequence participants were instructed to perform two tasks via the button press. The first task was to report the gender of the T1 face, while the second task was to judge T2 visibility so that participants made "yes or no" decisions whether or not they saw a face, and then rated their confidence how sure they were that the item was the face by using a 1-6 numerical keyboard scale (from low to high confidence). For each time lag between T1 and T2, detection signal trials comprised of 96 emotion-containing trials (happy or fearful faces), while detection noise trials comprised of 96 non-target-containing trials (scrambled faces). The hit rate was defined as the cumulative probability of reporting the emotional face given that an emotional face was the target ( $p$ ["emotion" | emotion]), and the false alarm rate was the cumulative probability of reporting an emotional face given that a scrambled face was the target ( $p$ ["emotion"। no emotion]). Given such categorization it was possible to compute the blink ROC that measured subject's ability to distinguish emotional faces from scrambled faces for T2 responses contingent on correct responses to $\mathrm{T} 1$. 
Figure 1.
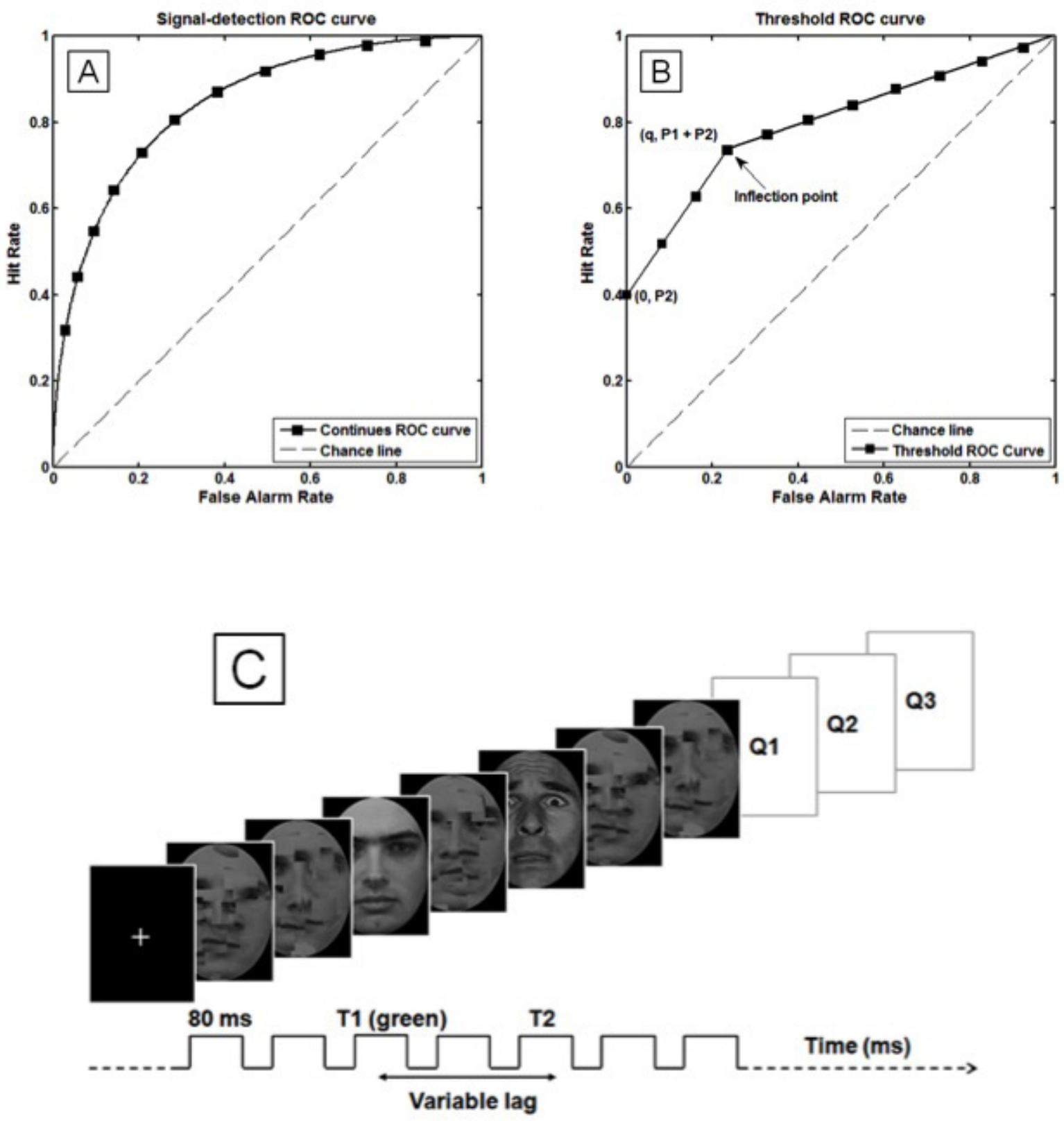

Note.

A) Continuous ROC curve predicted by signal-detection theory. Internal signal strength for making decisions is represented by Gaussian probability density functions. The false alarm rate is on the horizontal axis, and hit rate on the vertical axis.

B) Threshold ROC curve predicted by the three-state theory low- and high model. Internal signal strength for decision consistent with the 3-LHT has "all-or none" property containing thresholds. The point $(0, \mathrm{P} 2)$ is the origin of the lower limb. The limb's intersection is the point formed by the pair of false alarm q and the sum of the hit rates P1 and P2.

C) AB paradigm trial. In each trial, participants were asked to report gender of a neutral face (T1) presented in the stream of the 22-item RSVP (Q1). Then, participants had to report whether or not they saw a second T2-face (Q2), and rate their T2-visibility by using confidence judgments (Q3). T2 was presented in 50\% of all trials that were either happy or fearful facial expressions. Remaining RSVP items were distractors made by random rearrangement of neutral face features. T1 faces were presented within 8 and 12 item of the stream and had a greenish tint. T2 (if present) followed T1 by the lag of 2,3 or 4 items (160, 240 or 320 ms stimulus onset asynchrony). 
Figure 2.

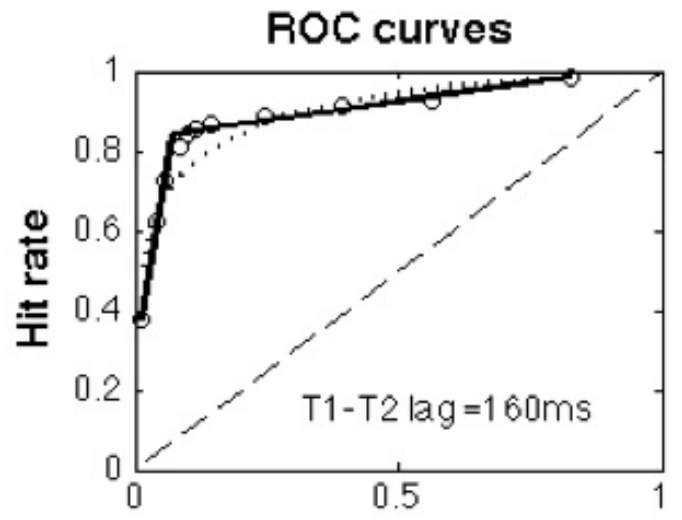

False alarm rate
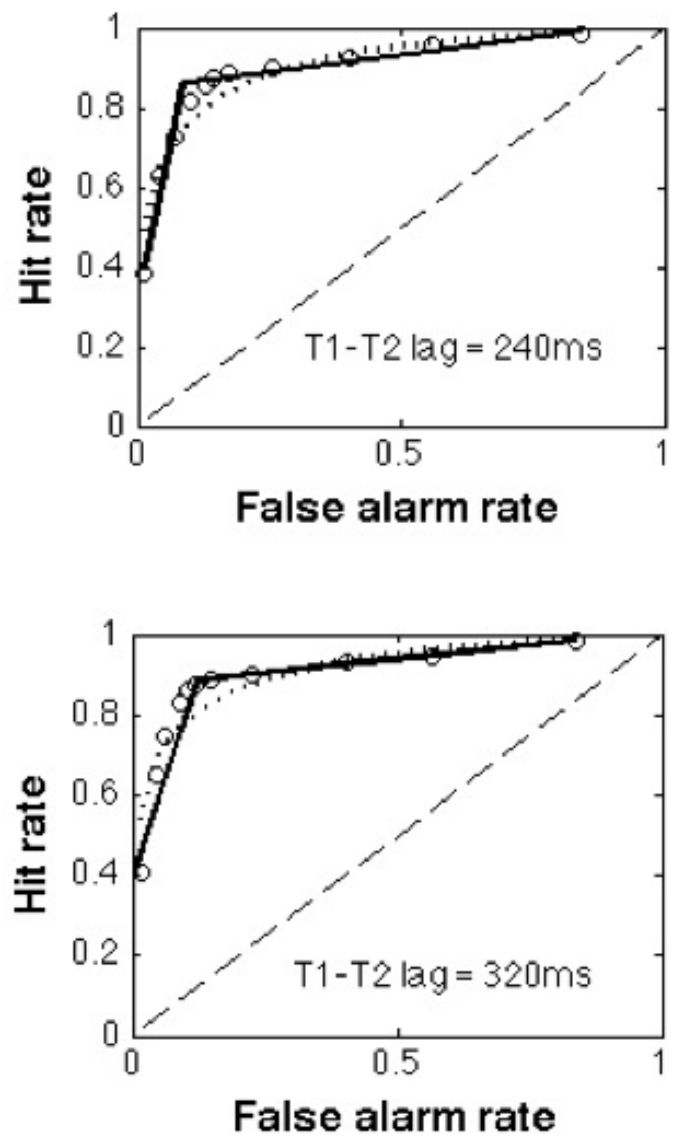

Note. Threshold and signal-detection ROC curves superimposed on the behavioral ROC data. Participants exhibited above-chance sensitivity at each lag.
A behavioral ROC curve examined the relationship between hits and false alarm rates at a number of different levels of response confidence (Yonelinas, Dobbins, Szymanski, Dhaliwali \& King, 1996). In particular, the ROC was generated by plotting pairs of the cumulative probabilities at subsequent confidence levels, corresponding to hit and false alarm rates (Macmillan \& Creelman, 2005). The cumulative data were the sums of proportions over confidence ratings, ranging in order from high confidence for emotional targets to high confidence in nonemotional targets. Finally, the ROC data were combined by pooling confidence judgments across all observers, and averaging their resulting signal-response matrices. To obtain a sensitivity measure of subject's ability to detect the emotional faces in the attentional blink condition, the area under the ROC ( $A^{\prime}$ ') was computed (Hanley \& McNeil, 1982). Participants were deemed aware when their A'values exceeded 0.5 , and the $95 \%$ confidence interval did not overlap with 0.5 .

A two-limb curve was fit for the given behavioral ROC data using the threshold model by Krantz (1969). Such ROC curve consists of a bottom limb from $(0, P 2)$ to $(\mathrm{q}, P 1$ $+P 2)$, and an upper one from $(\mathrm{q}, P 1+P 2)$ to $(1,1)$, where the $\mathrm{P} 1, \mathrm{P} 2$ are the probabilities of hits, and $\mathrm{q}$ is the probability of false alarms (see Fig.1B). To provide comparative fits for the threshold prediction, an unequal variance Gaussian signaldetection ROC curve was fit to the data (see Fig.1A) by estimating binormal parameters with the Rockit maximumlikelihood algorithm (Metz, Herman, \& Roe, 1998). To find the best-fit threshold ROCs, the confidence data were submitted to the Levenberg-Marquardt non-linear leastsquare algorithm (Press, Teukolsky, Vetterling, \& Flannery, 2002), respectively. Because one more free parameter of the more complex model (threshold model with tree parameters) could produce better fits than the simpler model (the signal-detection model with two free parameters), the quality of the fits was inspected with the F-test estimating the uncertainty in the model parameters (Yonelinas, Dobbins, Szymanski, Dhaliwali \& King, 1996) as well as the Akaike's Information Criterion with a second-order bias correction (AICc) adjusting for the number of free model parameters (Akaike, 1973; Burnham \& Anderson, 2004). In case of the F-test, a p-value less than the level of 0.05 indicated that the threshold model provided a better fit than the signal-detection model. In case of the AICc criterion, the winning model was the one with the lowest criterion value. Since the present experiment used the response criteria for twelve confidence levels, both goodness-of-fit measures considered additional 11 data points of the ROC curve.

\section{RESULTS}

We first calculated behavioral ROC curves to indicate whether or not there was a blink effect in case of discriminating $\mathrm{T} 2$. The behavioral group ROCs for all T1-T2 temporal lags are shown in Fig.2. In addition, the best threshold (solid line) and SDT (dashed line) fits were superimposed on the behavioral ROC curve as is shown in the figure. The sensitivity for blink ROC curves indicated 
above-chance performance (see Fig.2 for the A'). The sensitivity measures A' yielded the significant values above the level of $0.5: .899$ with $95 \% \mathrm{CI}(.844, .956)$ for the T1-T2 lag of $160 \mathrm{~ms}, .901$ with $95 \% \mathrm{CI}(.857, .960)$ for $240-\mathrm{ms}$ T1-T2 lag, and .911 with $95 \%$ CI $(.859, .964)$ for the $360-\mathrm{ms}$ T1-T2 lag. The A' results implicated that there was no blink effect involved in participants' performance at any lag, and in fact our experiment replicated a lack of AB outcomes for emotional faces that were presented by work of Milders and colleagues (2006).

The next step of our investigation was to compare the goodness-of-fits for both models. The determination coefficient measures indicated that the threshold ROC curve explained more of the variance in the data, $99.4 \%$, $97.8 \%, 99.3 \%$, than did the curvilinear ROC computed by the ROCKIT software, because their corresponding determination coefficients were lower for all T1-T2 lags, $91.0 \%, 84.9 \%$ and $87,2 \%$ correspondingly. A further analysis of the group ROC with the goodness-of-fit measures based on the F-statistics indicated that the threshold model was also found the best in modeling the attentional blink as compared to the standard signal-detection model, because for all lags the F-test gave the significant results in favor of the threshold curve, $F(1,8)=180.29$ for 160 -ms T1-T2 lag, $F(1,8)=21.10$ for $240-\mathrm{ms}$ lag, and $F(1,8)=92.94$ for $320-\mathrm{ms}$ lag. We also evaluated the information criteria AICc for the group data that indicated that the threshold model fit led to lower values of AICc, $-92.26,-76.13$ and -90.08 as compared to the signal-detection model which provided the following criteria values $-57.44,-63.18$ and -58.72 . To estimate the significance of such AICc comparisons, we averaged differences in the AICc data for the threshold and curvilinear ROCs for each subject. The mean $\triangle \mathrm{AICc}$ differences indicated the threshold model as the winning one for all T1-T2 lags: $\triangle \mathrm{AICc}=-27.78, t(22)=-4.02$ for the $160-\mathrm{ms}$ lag, $\triangle \mathrm{AICc}=-16.98, t(22)=-2.69$ for the 240 $\mathrm{ms}$ lag, and $\triangle \mathrm{AICc}=-18.2, t(22)=-3.40$ for the $320-\mathrm{ms}$ lag. In addition, we also investigated whether the thresholdlike processing had an advantage over the signal-detection model by inspecting SSE terms computed for both types of the ROC curve for each subject as recommended elsewhere (Yonelinas, Dobbins, Szymanski, Dhaliwali \& King, 1996). We compared the SEE terms both models with a Wilcoxon signed rank test, which indicated that the threshold model provided better fits than signal detection-model for all T1T2 lags, $W=81.0, W=79.0$ and $W=89.0(p<.001$ for all conditions). Taken together, the goodness-of-fit measures indicated that the threshold model produced better fits than predictions from the Gaussian model indicating that the function relating activation strength of emotion to awareness was in fact discrete.

\section{DISCUSSION}

The present results show that the $\mathrm{AB}$ data were better described by the two-limb ROC than the curvilinear ROC curve for all T1-T2 lag conditions. Thus, the present study contradicts activation strength hypothesis (Farah, 1994) that awareness of perceptual representation is a simple monotonic increasing function of the strength of the underlying representation. It suggests the thresholdlike quality of emotional representation has to do with the assignment of access priority to emotionally meaningful events. In this fashion, our findings are in line with Sergent and Dehaene (2004) who identified the "all-or-none" character of conscious perception in the attentional blink task. Those authors, however, used only a simple regression model to examine bimodality of the confidence data. Our study extends their view on awareness by providing evidence that perceptual information can be supported by psychophysical thresholds in order to be consciously accessed.

To date, there are two plausible explanations why detection of emotional meaning in the attentional blink task is aware. Most researchers agree that the twostage model (Chun \& Potter, 1995) gives the satisfactory explanation on the $\mathrm{AB}$ results with emotional targets. At the first stage, $\mathrm{T} 1$ and $\mathrm{T} 2$ are processed to a stage where features and even meaning are registered but they do not reach the level sufficient for conscious reports. Because the second stage can deal with only one target a time, T2 fail to enter awareness when the second stage is occupied by T1. Since the emotional meaning of stimuli is evaluated as being important they have preferential access to limitedcapacity processes crucial for short-term consolidation and awareness (Milders et al., 2006). The alternative account suggests that emotionally meaningful stimuli (for instance, one's own name or a signal of danger, etc.) are of higher saliency and therefore stronger activation of their representation is not affected by the ongoing processing of T1 (Shapiro, Caldwell, \& Sorensen, 1997). In conjunction with this account we propose that increased saliency of emotional items is associated with the threshold-like quality of that representation. Here, preferential access to processing of emotional T2 is supported by thresholds posited as distances between internal sensory states of the rectangular distributions of emotional targets.

\section{REFERENCES}

Akaike, H. (1973). Information Theory and an Extension of the Maximum Likelihood Principle. In B. N. Petrov \& F. Csaki, (Eds.). Second International Symposium on Information Theory (p. 267-281). Budapest: Akademiai Kaido.

Burnham, K. P., \& Anderson, D. R. (2004). Multimodel inference. Sociological methods and research, 33, 261-304.

Chun, M. M., \& Potter, M. C. (1995). A two-stage model for multiple target detection in rapid serial visual presentation. Journal of experimental psychology. Human perception and performance, 21(1), 109-127.

Ekman, P., \& Friesen, W. V. (1976). Pictures of facial affect. Palo Alto, CA: Consulting Psychologists Press.

Farah, M. J. (1994). Visual perception and visual awareness after brain damage: a tutorial overview. In C. Umilta. \& M. Moscovitch, (Eds.) Attention and performance, $X V$. Cambridge, MA: MIT Press.

Green, D. M., \& Swets, J. A. (1966). Signal Detection Theory and Psychophysics. New York: Wiley.

Hanley, J. A., \& McNeil, B. J. (1982). The meaning and use of the area under a Receiver Operating Characteristic (ROC) Curve. Radiology, 143(1), 29-36.

Kanwisher, N. (2001). Neural events and perceptual awareness. Cognition, 79(1-2), 89-113.

Krantz, D. H. (1969). Threshold theories of signal detection. Psychological Review, 76(3), 308-324. doi:10.1037/h0027238 
Lundqvist, D., Flykt, A., \& Öhman, A. (1998). The Karolinska Directed Emotional Faces - KDEF, CD ROM from Department of Clinical Neuroscience, Psychology section, Karolinska Institutet, ISBN 91630-7164-9.

Macmillan, N. A., \& Creelman, C. D. (2005). Detection theory: A user's guide. (2nd Edition). Mahwah, NJ: Erlbaum.

Maratos, F. A., Mogg, K., \& Bradley, B. P. (2008). Identification of angry faces in the attentional blink. Cognition \& emotion, 22(7), 13401352. doi:10.1080/02699930701774218

Metz, C. E., Herman, B. A., \& Roe, C. A. (1998). Statistical comparison of two ROC-curve estimates obtained from partially-paired datasets. Medical Decision Making, 18(1), 110-121.

Milders, M., Sahraie, A., Logan, S., \& Donnellon, N. (2006). Awareness of faces is modulated by their emotional meaning. Emotion, 6(1), 10-17. doi: 10.1037/1528-3542.6.1.10.

Phillips, M. L., Medford, N., Young, A. W., Williams, L., Williams, S. C., Bullmore, E. T., Brammer, M. J. (2001). Time courses of left and right amygdalar responses to fearful facial expressions. Human Brain Mapping, 12(4), 193-202

Press, W. H., Teukolsky, S. A., Vetterling, W. T., \& Flannery, B. P. (2002) Numerical Recipes. Cambridge, England: Cambridge University Press.

Schwarz, G. E. (1978). Estimating the dimension of a model. Annals of Statistics, 6(2), 461-464. doi:10.1214/aos/1176344136.

Sergent, S. C., \& Dehaene, S. (2004). Is Consciousness a Gradual Phenomenon. Psychological Science, 15(11), 720-728.

Shapiro, K. L., Caldwell, J., \& Sorensen, R. E. (1997). Personal names and the attentional blink: a visual "cocktail party" effect. Journa of experimental psychology. Human perception and performance, 23(2), 504-14.

Shapiro, K. L., Raymond, J. E., \& Arnell, K. M. (1997). The attentional blink. Trends in Cognitive Sciences, 1(8), 291-296.

Slotnick, S. D., \& Dodson, C. S. (2005). Support for a continuous (singleprocess) model of recognition memory and source memory. Memory \& Cognition, 33(1), 151-170

Stein, T., Peelen, M. V., Funk, J., \& Seidl, K. N. (2010). The fearfulface advantage is modulated by task demands: evidence from the attentional blink. Emotion, 10(1), 136-140. doi: 10.1037/a0017814.

Thomas, K. M., Drevets, W. C., Whalen, P. J., Eccard, C. H., Dahl, R. E., Ryan, N. D., \& Casey, B. J. (2001). Amygdala response to facia expressions in children and adults. Biological Psychiatry, 49(4), 309-316.

Yang, E., Zald, D. H., \& Blake, R. (2007). Fearful expressions gain preferential access to awareness during continuous flash suppression. Emotion, 7(4), 882-6. doi: 10.1037/1528-3542.7.4.882.

Yonelinas, A. P., Dobbins, I., Szymanski, M. D., Dhaliwal, H. S., \& King, L. (1996). Signal-detection, threshold, and dual-process models of recognition memory: ROCs and conscious recollection. Consciousness and Cognition, 5(4), 418-441.

\section{ACKNOWLEDGMENTS}

This research has been supported by the National Science Center (Poland), and funded under the grant's decision DEC-2011/03/B/HS6/01799 to R.S. 